\title{
2. Wiesbadener Symposium Kinderdermatologie ${ }^{1}$ Besonderheiten der kindlichen Hautbarriere und Konsequenzen für die pädiatrisch-dermatologische Praxis
}

\author{
2nd Wiesbaden Symposium Pediatric Dermatology \\ Specifics of Skin Barriere Function in Childhood and Consequences for the Dermopediatric Practice
}

Autor

Institut

\section{H. S. Müller}

HSM Kommunikations-Service im Gesundheitswesen, Wiesbaden

\section{Bibliografie}

DOI 10.1055/s-0028-1119686

Online-Publikation: 13.3. 2009

Akt Dermatol 2009; 35:

347-350 @ Georg Thieme Verlag KG Stuttgart · New York ISSN 0340-2541

Korrespondenzadresse Dipl.-Biol. Helmut S. Müller

HSM Kommunikations-Service im Gesundheitswesen

Forststraße 21

65193 Wiesbaden

info@hsm-pharmed.de
Die anatomischen und physiologischen Merkmale der Haut im Säuglings- und Kleinkindalter sowie deren Relevanz für Ätiologie, klinischen Verlauf und Therapie von Dermatosen bei Kindern verschiedener Altersgruppen standen im Mittelpunkt einer interdisziplinären dermato-pädiatrischen Fortbildungsveranstaltung, zu der die Klinik für Dermatologie und Allergologie gemeinsam mit der Klinik für Kinder und Jugendliche der HSK-Kliniken Wiesbaden eingeladen hatten. Praktische Tipps von Referenten und Teilnehmern lieferten wertvolle Informationen und „Handwerkliches“ für den dermatologischen und pädiatrischen Praxisalltag.

Schwerpunkte des kinderdermatologischen Austausches, zu dem sich rund 200 Kinderärzte und Dermatologen im Wiesbadener Kurhaus trafen, waren die Besonderheiten der kindlichen Haut und ihrer Barriereeigenschaften im Hinblick auf häufige Erkrankungen wie der Neurodermitis (๑ Abb. 1).

Hochkarätige Referenten beider Fachrichtungen vermittelten Hintergründe und wertvolle Tipps zu Diagnose und Behandlung von Hautkrankheiten, die im Säuglings- und Kleinkindalter für die Betroffenen und deren familiäres Umfeld besonders belastend sind.

Die Direktorin der Klinik für Dermatologie und Allergologie der HSK Wilhelm Fresenius Klinik Wiesbaden, Prof. Dr. Christiane Bayerl, betonte als Organisatorin, dass die Beschaffenheit des Organs Haut sich im Kindesalter permanent ändere, was im Praxisalltag stets zu beachten sei.

Der Mitorganisator Prof. Dr. Michael Albani, Direktor der Klinik für Kinder und Jugendliche der HSK Dr. Horst Schmidt Klinik Wiesbaden, zeigte sich erfreut über das große Interesse und die gleichgewichtige Beteiligung beider Fachgruppen. Es gelte, so Albani, beide Fachgebiete für die ${ }^{1}$ Mit freundlicher Unterstützung der Firma Stiefel Labo-
ratorium GmbH, Offenbach
Phänomene und Eigenheiten der kindlichen Haut zu sensibilisieren ( $\bullet$ Abb. 2).

\section{Epidermale Barriere bei Neugeborenen noch unterentwickelt $\nabla$}

Die Physiologie der Neugeborenenhaut, so der Kinderarzt und Dermatologe Prof. Dr. Peter Höger (Hamburg), sei von besonderer praktischer Relevanz für Therapie und Pflege. Postnatal sei die Vernetzung zwischen Dermis und Epidermis noch mangelhaft, die Haut dünner und die Barrierefunktion noch nicht voll ausgebildet. Zudem sei die Relation von Fläche zu Gewicht besonders groß und der Stoffwechsel noch nicht ausgereift. Folge sei, dass weit verbreitete, bei Erwachsenen oft harmlose Stoffe wesentlich leichter durch die Haut penetrieren und zu hohen Blutspiegeln bis hin zu Intoxikationen führen können. Die epidermale Unreife sei z.B. vielfach der Grund für Unverträglichkeitsreaktionen nach Anwendung von topischen Arzneimitteln (z.B. Neomycin, Salicylate) sowie häufig auch von ungeeigneten Pflegeprodukten. Beispielhaft nannte Höger die Verwendung von Harnstoffpräparaten bei trockener Haut. Unabhängig davon, dass trockene Hautzustände im Säuglingsalter aus den genannten Gründen ein „physiologisches Übergangsstadium“ seien, so Höger, rufe Harnstoff im Gegensatz zu Erwachsenen bei Säuglingen vielfach massive Hautreaktionen hervor. Als Feuchthaltefaktor empfahl er ersatzweise Glycerin und die Lokalanwendung von Nachtkerzenöl.

\section{„Intelligentere“ Externa gefordert $\checkmark$}

Ein weiteres Problem insbesondere im Kindesalter ist die Verwendung von Emulgatoren in Grundlagen topischer Zubereitungen. Sie können eine Emulgation und Auswaschung epidermaler 


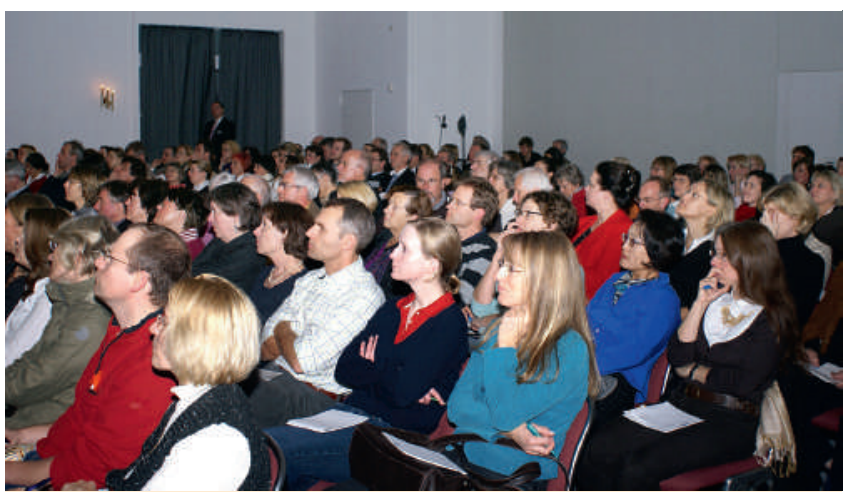

Abb. 1 Blick ins bis auf den letzten Platz besetzte Auditorium.

Lipide fördern und so die kindliche Hautbarriere weiter schwächen. „Wir brauchen intelligentere Externa mit körpereigenen Inhaltsstoffen“, so Höger. Statt der Verwendung von gängigen emulgatorhaltigen Cremes empfahl er bei Kleinkindern Präparate mit Zuckertensiden als Emulgatorersatz oder besser noch DMS-Cremes. Letztere enthalten Lipidkomponenten in sogenannter hautverwandter lamellarer Derma-Membran-Struktur $\left(D_{M}{ }^{\circledR}\right)$ und machen laut Höger Emulgatoren überflüssig. DMSCremes tragen so besonders zur Sicherheit und Unbedenklichkeit von Pflegeprodukten für die Anwendung im Kleinkindalter bei.

Für jede Art von externer Therapie und Pflege gelte der Grundsatz: Sicherheit und Unbedenklichkeit geht vor Wirksamkeit.

\section{Spezifische Infektionskrankheiten atopischer Haut $\nabla$}

Eine gestörte Hautbarriere, wie sie bei atopischer Diathese meist vorliegt, kann das Eindringen von Mikroorganismen erleichtern und dazu führen, dass zur normalen Mikroflora der gesunden Haut gehörende Bakterien und saprophytäre Pilze triggerartig eine Sensibilisierung, Immunreaktionen und Entzündungserscheinungen auslösen.

\section{Bakterien}

So verdeutlichte Prof. Dr. Helmut Schöfer (Frankfurt), dass eine Staphylococcus aureus-Sanierung im Kindesalter vielfach unumgänglich ist. S. aureus kolonisiert zwar die normale gesunde Haut und insbesondere auch die Nasenschleimhaut, bei entsprechender Prädisposition wie Immunerkrankungen oder bei atopischer Dermatitis weisen Staphylokokken jedoch ein anderes und intensiveres Besiedlungsmuster sowie eine deutlich erhöhte Besiedlungsdichte auf. Sie rufen Infektionen hervor wie Follikulitis, Impetigo, Furunkel bis hin zur Gangrän. Laut Schöfer gibt es eine Reihe von Gründen dafür, dass die atopische Haut für S. aureus empfänglicher ist, so die verminderte Barrierefunktion und eine eingeschränkte Funktion des angeborenen Immunsystems.

Bezüglich der Therapie verwies er auf die Leitlinien und Empfehlungen der Arbeitsgemeinschaft Dermatologische Infektiologie (ADI). Dabei sollte stets das Risiko der steigenden Resistenzentwicklung, die Sensibilisierung und das Effektivitätsmuster beachtet werden.

\section{Pilze}

Zur residenten Mikroflora der Haut gehört auch der saprophytäre Pilz Malaszezzia furfur, der sich insbesondere in lipidreichen Arealen wie im Hals- und Nackenbereich sowie auf der behaarten

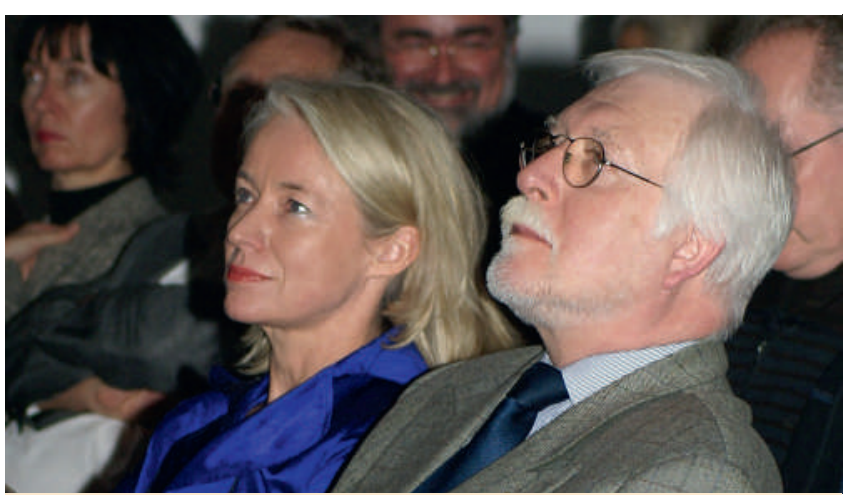

Abb. 2 Die Organistoren der interdisziplinären Veranstaltung Kinderdermatologie, Prof. Dr. Christiane Bayerl und Prof. Dr. Michael Albani.

Kopfhaut ansiedelt. Er kann Hauterkrankungen wie Pityriasis versicolor, seborrhoisches Ekzem, Follikulitis und auch das Atopische Ekzem begünstigen. Prof. Dr. Peter Mayser (Gießen) stützte in seinem Vortrag die Hypothese, dass durch die gestörte Hautbarriere die Penetration von Malaszezzia bei Neurodermitis erleichtert wird und der Saprophyt als Triggerfaktor und wichtiges Antigen allergische Reaktionen vom Soforttyp als auch vom Spättyp auslösen kann. Insbesondere Neurodermitis-Patienten vom „Head and neck-Typ“ haben im Nackenbereich eine erhöhte Besiedlung mit Malaszezzia. Kinder weisen ein deutlich höheres Sensibilisierungsrisiko auf. Anhand des Prick-Tests und auch des Atopie-Patch-Tests konnten Korrelationen zwischen Malaszezzia-spezifischen IgE-Werten und lokalen Hauterscheinungen nachgewiesen werden. Bei entsprechenden Befunden ist eine konsequente antimykotische Therapie angezeigt.

Viren

Patienten mit atopischem Ekzem neigen nach Angaben von Prof. Dr. Andreas Wollenberg (München) aufgrund eingeschränkter Immunfunktionen zu viralen Infektionen der Haut. Das Eczema herpeticatum definiert sich als flächige Superinfektion mit Herpes-simplex-Viren vom Typ 1, die sich auf die Grunderkrankung, die atopische Dermatitis, aufpfropft. Das Krankheitsbild, das sich in Form von disseminiert auftretenden, monomorphen vesikuloerosiven Eruptionen auf ekzematöser Haut präsentiert, zählt zu den schweren Komplikationen der atopischen Dermatitis. Meist tritt es an Kopf (insbesondere Augenregion) und Hals auf und ist mit schweren Allgemeinsymptomen wie Abgeschlagenheit, Fieber und Schmerzen assoziiert. Diagnostisch lässt sich das Eczema herpeticatum u.a. durch elektronenoptische Virusdirektnachweise und serologische Untersuchungen gut verifizieren. Therapeutisch bietet sich eine systemische Virusbehandlung an, die je nach Ausprägung durch entzündungshemmende Maßnahmen wie topische Steroide unterstützt werden kann.

\section{Quecksilberintoxikation bei Kindern \\ $\nabla$}

Prof. Dr. Christiane Bayerl berichtete über Quecksilberintoxikationen bei zwei Migrantenfamilien aus Kosovo-Albanien. Mutter und Kinder wiesen Symptome akuter und chronischer Intoxikationen auf, worauf hohe Quecksilberkonzentrationen in Blut und Urin nachgewiesen werden konnten. Nach Durchführung von Sanierungsmaßnahmen und einer Ausleitungstherapie waren die Patienten symptomfrei. Als Ursache und Quecksilberquelle erwiesen sich quecksilberhaltige Bleichsalben, die von den Müt- 


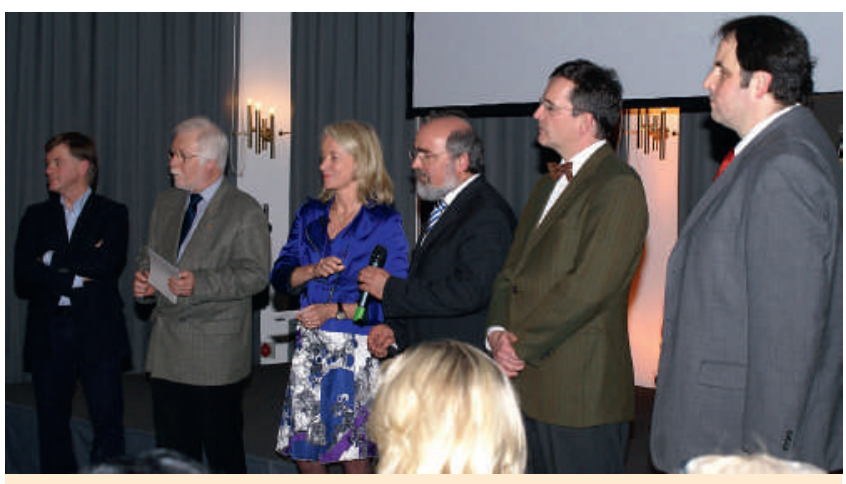

Abb. 3 Diskussion mit den Referenten nach dem ersten Abschnitt der Vorträge. Von links beginnend Prof. Dr. Peter Höger, Prof. Michael Albani, Prof. Christiane Bayerl, Prof. Helmut Schöfer, Prof. Dr. Andreas Wollenberg und Prof. Dr. Peter Mayser.

tern benutzt und offensichtlich über direkten Hautkontakt, beim Zubereiten der Nahrungsmittel durch die Mütter und durch Lagerung der Creme im Kühlschrank und Ausdünsten zur Kontamination der Kinder führten. In Diskussionbeiträgen wurde bestätigt, dass auch in afrikanischen Ländern immer noch schwermetallhaltige Kosmetika, insbesondere Bleichsalben Verwendung finden, die zu massiven Intoxikationen führen können (๑ Abb.3).

\section{Hautbiopsien im Kleinkindesalter \\ $\nabla$}

Als sicherstes Mittel zur Beschreibung von Hauterkrankungen stellt die Hautbiopsie den Goldstandard bei der Erkennung von Differenzialdiagnosen dar. Diese Routinemethode, die von nahezu allen Dermatologen genutzt wird, hat bei den Kinderärzten bisher nicht die ihr zustehende Anerkennung gefunden. PD Dr. Wolfgang Weyers (Freiburg) zeigte eindrucksvoll, wie anhand des Korrelats zwischen klinischem Bild und histologischem Befund sichere Differenzialdiagnosen gestellt werden können. In schwierigen Fällen, so Weyers, gleiche die Diagnosestellung ohne Hautbiopsie einem „Blindflug“ ( $\bullet$ Abb.4).

\section{Kawasaki-Syndrom}

$\nabla$

Prof. Dr. Michael Albani gab ein Update zum Kawasaki-Syndrom, das vor allem bei Kindern in den ersten 2 bis 5 Lebensjahren auftritt. Es ist die häufigste systemische Vaskulitis. Sie verläuft im akuten Stadium hoch fieberhaft (5-10 Tage) mit deutlicher Antibiotikaresistenz. Weitere Symptome sind ein feinflächiges multiformes Exanthem, diffuse Lymphknotenschwellung, Himbeerzunge, Lacklippen, Konjunktivitis und ein Palmarerythem mit Hautabschilferungen. Häufig treten auch multiforme Organbeteiligungen, insbesondere Koronaraneurysmen auf. Eine frühe Erkennung, Diagnostik und Therapieeinleitung ist wichtig, um Komplikationen wie z.B. massive Koronaraneurysmen zu vermeiden. Therapeutisch ist das schwere Krankheitsbild mit Immunglobulinen i. V. und begleitend ASS gut beherrschbar.

\section{Akne im Säuglings- und Kindesalter $\nabla$}

Akne, die weltweit häufigste Hauterkrankung, kann in jedem Lebensalter, auch in allen Kindesalterstufen auftreten. Als präpu-

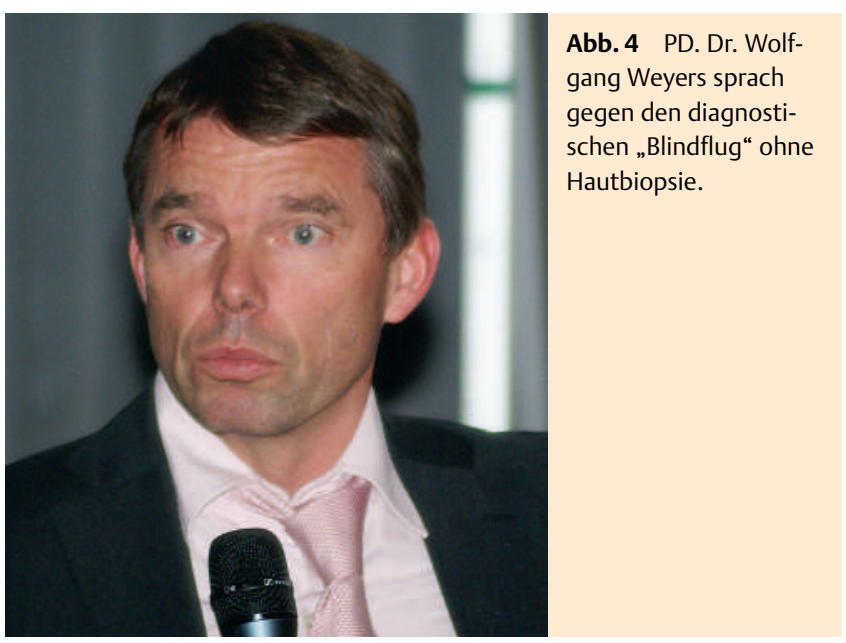

bertäre Akneformen, so PD Dr. Thomas Jansen (Essen), seien die häufigere, oft spontan abheilende Acne neonatum und die seltenere, aber schwerer verlaufende Acne infantum abzugrenzen. Der Referent gab einen aktuellen Überblick über die hormonalen Zusammenhänge insbesondere bei der Säuglingsakne. Die äußerliche Therapie - sofern erforderlich und gewünscht - erfolgt ähnlich wie beim Jugendlichen z. B. mit Fixkombinationen wie Clindamycin + Benzoylperoxid (Duac ${ }^{\circledR}$ Akne-Gel). Säuglinge vertragen dieses Kombinationsexternum gleich gut wie Jugendliche und Erwachsene. Schwere Fälle, insbesondere bei der Acne infantum, erfordern oft eine innerliche Therapie ( $\bullet$ Abb.5).

\section{Juckreiz: Chronifizierung unbedingt vermeiden}

Pruritus ist ein oft unterschätztes Symptom, das gerade im Kindesalter zu besonderen Belastungen der Betroffenen und des familiären Umfeldes führen kann. Prof. Dr. Sonja Ständer (Münster) stellte neue Erkenntnisse zu Juckreiz und Kratzverhalten im Kindesalter vor. Auch wenn zielgerichtetes Kratzen erst ab dem 2. Lebensjahr und eine detaillierte Differenzierung der Juckreizzustände erst nach etwa dem 6 . Lebensjahr zu erwarten ist, reagieren Neugeborene ab dem 2 Lebensmonat auf Pruritus-Impulse. Das sensorische Nervensystem ist schon ab der Geburt komplett ausgebildet und es laufen die gleichen Mechanismen ab wie bei größeren Kindern. Beteiligt sind Neurorezeptoren und Nervenfasern mit Neuropeptidausschüttung, die eine Reizleitung zum Gehirn gewährleisten. Anschließend kommt es zur Auslösung des Kratzreflexes, der sich bei Säuglingen meist in Form von Kneifen äußert ( $\bullet$ Abb. 6).

Pruritus kann auch an klinisch erscheinungsfreier Haut auftreten, sodass auch in diesen Fällen im Hinblick auf die mögliche Chronifizierung Therapiemaßnahmen zu treffen sind. Neben verschiedenen systemischen Erkrankungen (z.B. Leber, Niere) ist der Pruritus Kernsymptom von Hauterkrankungen, insbesondere des atopischen Ekzems. Das Motto bei Kindern, so Ständer, sei die frühzeitige effiziente Behandlung von Juckreiz, um eine Chronifizierung zu verhindern. Neben den „Erste-Hilfe-Maßnahmen“ wie einer rückfettende Basistherapie, Juckreizlinderung über Kühlung mit Menthol oder Campher, bei älteren Kindern Harnstoff und Lokalanästhetika könnten Corticoide, Capsaicin und Calcineurininhibitoren zum Einsatz kommen. Ständer verwies auf die juckreizhemmende Wirkung von Cannabinoidrezeptor-Agonisten und die guten Erfolge in entsprechenden $\mathrm{Zu}$ bereitungen. 


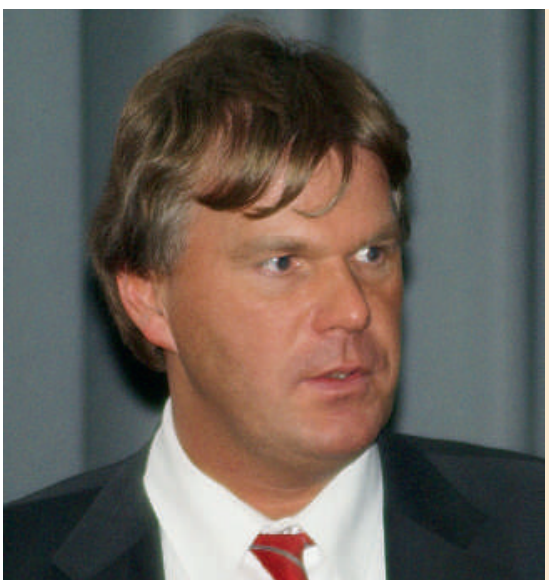

Abb. 5 Zur Lokaltherapie im Säuglingsund Jugendalter sprach PD Dr. Thomas Jansen.

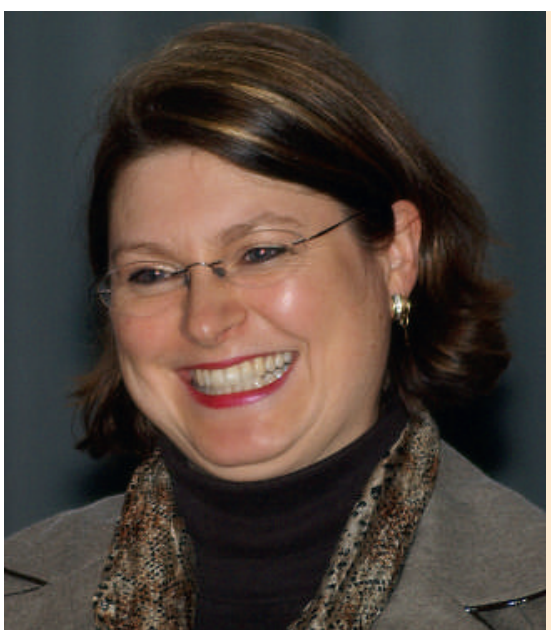

Abb. 6 Kneifen sich Säuglinge, ist dies der erste Hinweis, dass Juckreiz empfunden wird, berichtete Prof. Dr. Sonja Ständer.

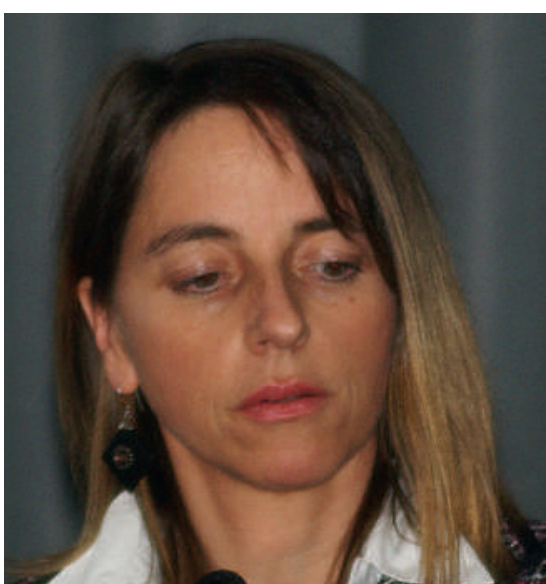

Abb. 7 Prof. Dr. Bernadette Eberlein stellte die Ergebnisse der Neurodermitis-Behandlung mit N-Palmitoylethanolamin vor.

\section{ATOPA: Wirknachweise überzeugen}

$\nabla$

Prof. Dr. Bernadette Eberlein (München) stellte die Ergebnisse einer multizentrischen Studie an mehr als 2400 Patienten mit atopischer Dermatitis (darunter über 900 Kinder) vor. Sie betonte, dass die Hautpflege und Basistherapie zur Wiederherstellung einer funktionierenden Hautbarriere ein Grundpfeiler der Neurodermitis-Behandlung sei. Im Verlauf der ATOPA-Studie erwies sich ein N-Palmitoylethanolamin (PEA)-haltiges Pflegepräparat als sehr effizient und gut verträglich. Alle Symptome wie Juckreiz, Trockenheit, Erythem, Schuppung, Exkoriation und Lichenifikation konnten signifikant reduziert werden. Die Schlafqualität verbesserte sich im Verlauf der Studie ebenfalls signifikant und der Einsatz von Kortikoiden konnte deutlich gesenkt werden. Kinder sprachen auf die konsequente Pflege sogar noch besser an als Erwachsene, wobei insbesondere der Juckreiz schon nach kurzer Zeit deutlich reduziert wurde ( $\bullet$ Abb. 7).

Der antipruritische Effekt von PEA beruht u.a. auf der Hemmung der Histaminausschüttung sowie der Aktivierung von Rezeptoren auf Juckreiz leitenden Neuronen, wodurch die Reizleitung unterbunden wird.

\section{Filaggrin und atopische Dermatitis $\nabla$}

Prof. Dr. Thomas Schwarz (Kiel) präsentierte neue Erkenntnisse zur Bedeutung von Filaggrin-Gendefekten für die Ätiologie der atopischen Dermatitis. Filaggrin spielt bei der zellulären Vernetzung im Statum corneum eine wichtige Rolle und trägt wesent-

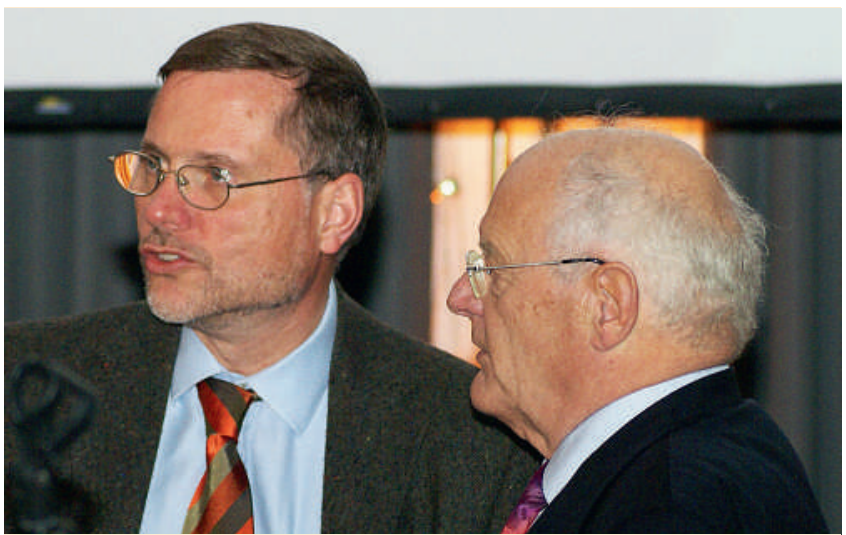

Abb. 8 Prof. Dr. Thomas Schwarz in der Pausendiskussion mit Prof. em. Dr. Ernst G. Jung.

lich zum Aufbau, zur Stabilität und zum Erhalt der epidermalen Strukturen bei. „Deshalb überrascht es nicht“, so Schwarz, „dass genetische Mutationsdefekte massiv in die Barrierefunktion eingreifen und morphologisch ichthyosisartige Erscheinungsformen hervorrufen können“. Patienten mit Filaggrinmutationen haben nicht nur ein erhöhtes Risiko zum atopischen Ekzem, sondern haben auch eine stärkere Neigung zu allergischen Reaktionen bis hin zur allergischen Rhinitis und erhöhtem Risiko zu Asthma bronchiale.

Man gehe heute davon aus, so Schwarz, dass neben dem sensibilisierten Immunsystem und umweltbedingten Triggerfaktoren der Barrieredefekt der Haut ein wichtiger Primärfaktor für die atopische Dermatitis ist. Eine therapeutische Konsequenz seien deshalb frühzeitige Maßnahmen zur Rekonstitution der epidermalen Barriere.

\section{Fazit der Veranstaltung \\ $\nabla$}

Die Vorträge wurden durch Diskussionsbeiträge der Experten und rege Beteiligung des Auditoriums ergänzt. Vielfältige eigene Erfahrungen und Tipps trugen zur Praxisnähe bei.

Die Referenten und Teilnehmer, die auch die Kaffeepause und den anschließenden Stehempfang zum regen Erfahrungsaustausch nutzten ( $\bullet$ Abb. 8), waren sich einig, dass die künftig alle zwei Jahre geplante Fortbildung großen praktischen Wert habe und zur notwendigen Sensibilisierung interdisziplinärer Betrachtungsweisen kindlicher Dermatosen beitrage. 